\title{
A NOTE ON MOUFANG-LIE RINGS
}

\section{ERWIN KLEINFELD ${ }^{1}$}

Malcev [2] has defined a ring to be Moufang-Lie in case it has the properties that (i) $x^{2}=0$, and (ii) $(x y, y, z)=y(x, y, z)$, for all elements $x, y, z$ of the ring, where the Jacobian $(x, y, z)$ is defined by $(x, y, z)$ $=(x y) z+(y z) x+(z x) y$. Any alternative ring under a new product $(x, y)=x y-y x$ is a Moufang-Lie ring. The main result of this note is the following:

Theorem. If a Moufang-Lie ring $R$, of characteristic different from 2 , contains an element a such that $a R=R$, then $R$ is a Lie ring.

In any anti-commutative ring $(x, y, z)$ may be verified to be a skewsymmetric function in three variables. That is to say it is zero if any two of the variables are equal, is left unchanged by an even permutation and is changed in sign by an odd permutation of the three variables. Another fact which may be verified to hold in any anti-commutative ring is the identity

$$
\begin{aligned}
w(x, y, z)-x(y, z, w) & +y(z, w, x)-z(w, x, y) \\
= & (w x, y, z)+(y z, w, x)+(w y, z, x)+(z x, w, y) \\
& -(z w, x, y)-(x y, z, w) .
\end{aligned}
$$

From this point on we assume that all elements lie in a Moufang-Lie ring of characteristic different from 2. We define the analogue of $f(w, x, y, z)$, previously defined in an alternative ring [1], by means of

$$
f(w, x, y, z)=(w x, y, z)-x(w, y, z)-(x, y, z) w .
$$

Then it follows from (2) and the skew-symmetry of the Jacobian that $f(w, x, y, y)=0$. On the other hand $f(x, y, y, z)=0$ follows from (ii) and the skew-symmetry of the Jacobian. Finally $f(x, x, y, z)=0$ follows from (i). This suffices to establish the skew-symmetry of $f(w, x, y, z)$ as a function of four variables. The identity

(3) $g(w, x, y, z)=(w x, y, z)+(x y, z, w)+(y z, w, x)+(z w, x, y)=0$, can be proved by applying $f(w, x, y, z)$ to each of the four terms of (3), together with the skew-symmetry of $f(w, x, y, z)$. By adding $g(w, x, y, z)-g(x, w, y, z)$ to the right hand side of (1) we obtain

Received by the editors May $17,1957$.

1 This work was supported in part by the Office of Naval Research under contract ONR-609(19) with Yale University. 


$$
\begin{aligned}
w(x, y, z)-x(y, z, w)+y(z, w, x)- & z(w, x, y) \\
& =3(w x, y, z)+3(y z, w, x) .
\end{aligned}
$$

On the other hand

$$
\begin{aligned}
2 f(w, x, y, z)-w(x, y, z)+x(y, z, w)- & y(z, w, x)+z(w, x, y) \\
& =(w x, y, z)+(y z, w, x),
\end{aligned}
$$

may be verified by applying $f(w, x, y, z)$ to each of the two terms on the right hand side of (5). Adding (4) and (5) together results in $2 f(w, x, y, z)=4(w x, y, z)+4(y z, w, x)$, so that

$$
f(w, x, y, z)=2(w x, y, z)+2(y z, w, x) .
$$

At this point we are ready to prove the theorem. There must exist an element $b$ such that $a b=a$. From the skew-symmetry of the Jacobian it follows that $(x, a, a b)=0$, for every element $x$ of $R$. But $(x, a, a b)=(x, a, b) a=(a x, a, b)$, using (2) and the skew-symmetry of $f(w, x, y, z)$. Hence $(a x, a, b)=0$. But in view of the hypothesis this means $(y, a, b)=0$, for all elements $y$ in $R$. Then $(y z, a, b)=0$ implies $f(y, z, a, b)=0$, for all $y, z$ in $R$, because of (2). As a consequence of (6), $2(y, z, a b)=0$ follows, so that $(y, z, a b)=0$. However $a b=a$, hence $(y, z, a)=0$. But then for all elements $x, y, z$ in $R,(x y, z, a)=0$. Again because of (2) it follows that $f(x, y, z, a)=0$, and then $f(a, x, y, z)=0$. Consequently $2(a x, y, z)=0$, as a result of (6). But then $(a x, y, z)=0$. Since $a R=R$ this means all Jacobians of $R$ are zero, so that $R$ is a Lie ring and the theorem is proved.

A number of remarks are in order. A result completely analogous to the above theorem holds for alternative rings. That is if $a$ is an element of an alternative ring with the property that $(a, R)=R$, then $R$ must be associative. No restriction on the characteristic is assumed this time. The proof consists of deriving exact analogues of the identities developed here. One may ask whether the conditions $(a, R)$ $=R$, and $a R=R$ can be fulfilled in an associative ring and Lie ring respectively. The answer is in the affirmative, provided we do not restrict ourselves to finite-dimensional algebras. Thus consider the free ring $S$ on two generators $x, y$ over a field of characteristic zero, $I$ the ideal generated by $x y-y x-1$. Then the differential ring $R$ $=S / I$ is simple and moreover $(x, R)=R$. Of course the Lie ring associated with $R$ satisfies the condition $x R=R$. It seems to be an open question however whether there exists an associative ring $R$ with the property that for every $a \neq 0,(a, R)=R$. Such a ring would of necessity have to be simple, without center and without divisors of zero. 


\section{ERWIN KLEINFELD}

\section{BIBLIOGRAPHY}

1. R. H. Bruck and Erwin Kleinfeld, The structure of alternative division rings, Proc. Amer. Math. Soc. vol. 2 (1951) pp. 878-890.

2. A. I. Malcev, Analytic loops, Rec. Math. (Mat. Sbornik N.S.) vol. 36 (78) (1955) pp. 569-576 (Russian).

\section{Yale UNIVERSity}

